\title{
Modulation of Gap Junctional Mechanisms during Calcium-Free Induced Field Burst Activity: A Possible Role for Electrotonic Coupling in Epileptogenesis
}

\author{
Jose L. Perez-Velazquez, Taufik A. Valiante, and Peter L. Carlen \\ Playfair Neuroscience Unit, Toronto Hospital Research Institute, Bloorview Epilepsy Program and Department of \\ Physiology, University of Toronto, Toronto, Ontario, Canada M5T 2S8
}

To date, there is little experimental evidence supporting or refuting electrotonic interactions through gap junctions in the generation and/or spread of seizure activity in the mammalian brain. We have studied gap junctional mechanisms in the in vitro calcium-free induced model of epilepsy using electrophysiological and staining techniques in the CA1 area of the hippocampus. Lucifer yellow staining of CA1 pyramidal neurons revealed that dye coupling was increased 2.3 times in hippocampal slices made hyperexcitable by perfusion with calcium-free artificial cerebrospinal fluid (aCSF). Furthermore, multiple neuronal dye coupling (triplets, quintuplets) was observed in these conditions but never in control (standard aCSF). Under conditions that reduce gap junctional conductance (intracellular acidification, octanol, halothane), seizure-like activity was suppressed in the CA1 area in this epilepsy model, whereas increasing gap junctional conductance by intracellular alkalinization increased the frequency and duration of field burst events. Intracellular acidification also reduced dye coupling as well as the frequency of fast prepotentials (electrotonic potentials) without altering neuronal firing frequency. Simultaneous extracellular field and single whole-cell recordings revealed suppression of synchronization between neuronal firing and spontaneous field burst activity during acidification. These observations indicate an apparent increase in electrotonic coupling during calcium-free induced spontaneous rhythmic field burst activity in the CA1 area of the hippocampus and that electrotonic coupling may contribute substantially to the synchronization of neuronal firing underlying seizure-like events.

[Key words: epilepsy, electrotonic coupling, gap junctions, hippocampus, field potential, whole-cell recordings]

The cellular bases of neuronal firing synchronization, a hallmark of epilepsy, have been the subject of extensive investigation. Much debate has dealt with the possible mechanisms for synchronizing the firing of large neuronal populations in the second

Received Sept. 28, 1993; revised Dec. 20, 1993; accepted Jan 13, 1994.

This work was supported by a grant from the Medical Research Council of Canada (MRC) to P.L.C. T.A.V. is an MRC student and J.L.P.-V. is a recipient of a NATO research fellowship. We thank Drs. Linda Mills and Muhammad Abdul-Ghani for their help.

Correspondence should be addressed to Dr. Peter L. Carlen, Playfair Neuroscience Unit, Toronto Western Hospital, 399 Bathurst Street, Toronto, Ontario M5T 2S8, Canada.

Copyright (C) 1994 Society for Neuroscience $0270-6474 / 94 / 144308-10 \$ 05.00 / 0$ and millisecond time scales. It is thought that electrical interactions, changes in extracellular ion concentrations, and excitatory feedback are necessary for synchronization (Taylor and Dudek, 1982; Dudek et al., 1986; Traub et al., 1989). That synaptic excitatory feedback is not essential for synchronization was shown in a series of experiments that gave rise to the widely used model of low calcium induced epilepsy (Taylor and Dudek, 1982; Haas and Jefferys, 1984; Konnerth et al., 1986; Schweitzer et al., 1992). Several groups have shown that seizure-like activity characterized by negative-going baseline shifts in extracellular field potential recordings and spontaneous bursts of population spikes (which represent synchronized action potentials) occurs when synaptic transmission is blocked with calcium-free solutions. This spontaneous activity, or "field bursts," has been observed in the three main areas of the hippocampal formation. It thus appears that electrical interactions and changes in ion concentration alone seem to be able to synchronize the activity of a large population of neurons.

Electrical interactions can be divided into field effects (ephaptic transmission) and electrotonic coupling through gap junctions. Electrotonic interactions have been shown to occur in neural networks characterized by synchronized behavior (for a recent review on gap junctions, see Dermietzel and Spray, 1993). There is electrophysiological and anatomical evidence for the existence of gap junctions in the three main areas of the hippocampus, CA1, CA3, and dentate gyrus (MacVicar and Dudek, 1981, 1982; Schmalbruch and Janhsen, 1981; Taylor and Dudek, 1982; Dudek et al., 1983; Shiosaka et al., 1989; Nuñez et al., 1990; Baimbridge et al., 1991).

The role of electrotonic coupling in the generation of epileptiform activity has been dismissed on the basis of very restricted coupling between neurons (Haas and Jefferys, 1984). However, gap junctional conductance is not a static mechanism but a dynamic one controlled by several factors. Changes in junctional conductance may have profound effects on the coupling between cells, which will alter population dynamics. For example, the incidence of intercellular electrotonic coupling changes during development (Peinado et al., 1993). The degree of coupling is controlled mainly by intracellular pH (Spray et al., 1981, 1986; MacVicar and Jahnsen, 1985), although internal calcium also plays a role (Lazrac and Peracchia, 1993). Acidification is known to reduce gap junctional conductance and block electrotonic coupling, whereas alkalinization increases junctional conductance. The $\mathrm{pH}$ dependence of gap junctional conductance is very steep: at $\mathrm{pH} \leq 6.8$, gap junctions are completely blocked and have maximal conductance at $\mathrm{pH} \geq 7.8$. This modulation of 
conductance occurs rapidly in a time scale of seconds (Spray et al., 1981, 1986). Rapid changes in $\mathrm{pH}$ have been observed in the brain slice during epileptic activity and intense neuronal firing (Somjen, 1984; Walz, 1988; Chen and Chesler, 1992; Chesler and Kaila, 1992), specifically an initial alkalinization followed by a phase of acidification during paroxysmal discharges. The former is thought to be caused by accumulation of bicarbonate in the extracellular space and the latter due to lactic acid produced in neurons during periods of intense activity. Thus, gap junctional conductance could be altered continually during seizure activity in epilepsy, increased by alkalinization and decreased by acidification and, in particular, during field burst activity in the calcium-free model.

We investigated the possible role of electrotonic interactions through gap junctions in the CAl area of the rat hippocampus in the calcium-free model of epilepsy using electrophysiological and staining techniques to correlate neuronal coupling to cellular and population behavior. The spontaneous field burst activity of the hippocampal formation in the calcium-free model has all the characteristics of cortical and hippocampal epileptic activity observed in vivo. Since during seizure activity in the brain there is a decrease in extracellular calcium and an increase in potassium (Dichter, 1984), this in vitro model is very useful for analyzing cellular mechanisms of epileptiform discharges. Indeed, it provides a simplified system in which to study nonsynaptic interactions, and further supports the notion that synaptic transmission may only play a part in the generation of seizure activity in the intact brain. Coupling via gap junctions in the CA1 area was investigated by intracellular dialysis of Lucifer yellow using the patch-clamp technique, a dye that has been well characterized for measuring coupling via gap junctions (Stewart, 1981; Andrew et al., 1982; MacVicar and Dudek, 1982; Nuñez et al., 1990; Church and Baimbridge, 1991). To study effects of alterations of gap junctional conductances on the spontaneous field burst activity, the strength of electrotonic coupling was altered by well-characterized methods such as changes in intracellular $\mathrm{pH}$ and by adding octanol or halothane to the calcium-free aCSF. Acidification of the cellular cytoplasm, which blocks gap junctions, was expected to diminish spontaneous field bursting (and decrease the incidence of dye coupling), while alkalinization was expected to increase firing synchronization among neurons, therefore increasing the frequency and duration of field bursts.

\section{Materials and Methods}

Brain slices and solutions. Young Wistar rats (20-30 d old) were anesthetized with halothane (Fluothane, Ayerst Laboratories, Montreal) and decapitated. Transverse brain slices $(400 \mu \mathrm{m})$ were obtained using a Vibratome (Series 1000, Technical Products International, Inc.) and maintained in artificial cerebrospinal fluid (aCSF) that contained (in mm) NaCl, 125; KCl, 5; $\mathrm{NaH}_{2} \mathrm{PO}_{4}, 1.25 ; \mathrm{MgSO}_{4}, 2 ; \mathrm{CaCl}_{2}, 2 ; \mathrm{NaHCO}_{3}$, 25 , glucose, $10, \mathrm{pH} 7.4$ aerated with $95 \% \mathrm{O}_{2}, 5 \% \mathrm{CO}_{2}$. Calcium-free aCSF was the same with no added $\mathrm{CaCl}_{2}$ and with 1 mM EGTA to reduce contaminant calcium levels. When required, $25 \mathrm{~mm} \mathrm{NaCl}$ was substituted by sodium propionate (for intracellular acidification), 10 mM $\mathrm{NaCl}$ by ammonium chloride $\left(\mathrm{NH}_{4} \mathrm{Cl}\right.$, for alkalinization and later acidification) or octanol $(0.2 \mathrm{~mm})$ was added. The $\mathrm{pH}$ of all solutions (kept at 7.4) was verified for each experiment. Similarly, changes in $\mathrm{NaHCO}_{3}$ concentration were balanced by an equimolar change in $\mathrm{NaCl}$. Osmolarity was $300 \pm 5$ mOsm. For whole-cell recordings, internal solution contained (in mM) K-gluconate, 150; HEPES, 10; Mg-ATP, 2; $\mathrm{KCl}, 5 ; \mathrm{pH} 7.2$ adjusted with $\mathrm{KOH}$ and osmolarity of $275 \pm 5 \mathrm{mOsm}$.

Electrophysiological recordings. For recordings, slices were transferred to a superfusion chamber maintained at $35^{\circ} \mathrm{C}$ (Medical System Corp., Model PDMI-2). Neuronal recordings were performed using the whole-
Table 1. Incidence of dye coupling between CA1 pyramidal neurons in control, during epileptiform activity, and during intracellular acidification

\begin{tabular}{lcl} 
& $\begin{array}{c}\text { Coupling } \\
\text { observed }\end{array}$ & $\begin{array}{l}\text { Percent- } \\
\text { age of } \\
\text { coupling }\end{array}$ \\
\hline Control aCSF $(n=33)$ & 5 & $15.1 \%$ \\
Calcium-free aCSF $(n=38)$ & 13 & $34.2 \%$ \\
Calcium-free/propionate $(n=23)$ & 1 & $4.3 \%$
\end{tabular}

Dye coupling is compared in standard aCSF and during field burst activity in calcium-free aCSF. Numbers represent instances when a recording showed two or more neurons dye coupled. Third line is coupling observed in calcium-free aCSF containing $25 \mathrm{~mm}$ sodium propionate. Lucifer yellow $(0.1 \%$ in internal solution) was dialyzed using patch-clamp recording electrodes for $30 \mathrm{~min}$. Results are significant with a $99 \%$ confidence (Student's $t$ test).

cell configuration of the patch-clamp technique (Hamill et al., 1981). Patch pipettes were pulled from borosilicate capillary tubing (World Precision Instruments, New Haven). Electrodes had tip resistances ranging from 4 to $6 \mathrm{M} \Omega$ when filled with the internal solution. The resistance to ground of the whole-cell seal was 3-8 G $\Omega$ before breaking though the membrane, and the series resistance was less than $20 \mathrm{M} \Omega$ after breakthrough. An agar bridge made with external solution was used to minimize junction potential. Neuronal responses were recorded using an Axoclamp $2 \mathrm{~A}$ amplifier. Extracellular field potentials were recorded using a patch pipette filled with $2 \mathrm{M} \mathrm{NaCl}$ (resistance was then 2-3 M $\Omega$ ), and recorded via an Axopatch $200 \mathrm{~A}$ amplifier. The recording electrode was always placed in the cell body layer. Both signals (neuronal and field recordings) were displayed on a chart recorder (Gould 2200), digitized at $88 \mathrm{kH}$., and stored on viden tape using a digital data recorder VR-10 (Instrutech Corp., NY) for later playback and analysis. Neuronal responses to intracellular current injection were generated and digitized using pCLAMP software (Axon Instruments, CA), through a TL-1 Labmaster analog-to-digital converter. For quantifying fast prepotentials (FPPs) and action potential frequency, 30-60 sec of neuronal activity was digitized $(10 \mathrm{kH} z)$ using Fetchex (Axun Instruments). FPPs and action potentials were detected automatically using a template recognition algorithm, and amplitudes binned in $0.5 \mathrm{mV}$ increments, using the TCRUNCH (Intelligent Software Solutions, Canada) software package. Events less than $0.5 \mathrm{mV}$ were discarded from the analysis. Whole-cell recordings and dye fillings were performed in neurons that had membrane potentials more negative than $-50 \mathrm{mV}$ and action potential amplitudes $>60 \mathrm{mV}$ in response to a square-wave depolarizing pulse.

Iucifer yellow staining. Neurons were dialyzed with internal solution containing $0.1 \%$ of Lucifer yellow (Lucifer yellow, dipotassium salt, Sigma Chemical Co., St. Louis, MO) for no less than $30 \mathrm{~min}$. Dye coupling was evaluated first at the end of the dialysis using a Nikon Labophot-2A/2 fluorescent microscope. Following dye dialysis, slices were fixed overnight at $4^{\circ} \mathrm{C}$ in $4 \%$ paraformaldehyde in isoosmotic aCSF, and then dehydrated in a graded ethanol series and cleared in methyl salicylate. Stained cells were then again evaluated using a Bio-Rad MRC 600 confocal microscope equipped with fluorescein isothiocyanate filters. Confocal micrographs were printed on a Kodak SV6500 video printer.

\section{Results}

\section{Dye coupling during spontaneous field burst activity}

Electrotonic interactions are a very dynamic mechanism of intercellular communication modulated mainly by cytoplasmic pH (Spray et al., 1981, 1986; MacVicar and Jahnsen, 1985). Since changes in $\mathrm{pH}$ have been reported to occur during periods of intense neuronal firing, we studied whether there was a change in neuronal coupling through gap junctions during field burst activity in the calcium-free model. To assess the incidence of coupling between CA1 pyramidal neurons, these were filled with Lucifer yellow, a very well characterized dye that crosses gap junctions (Stewart, 1981; Andrew et al., 1982). Dye coupling during the period of calcium-free induced field burst activity 

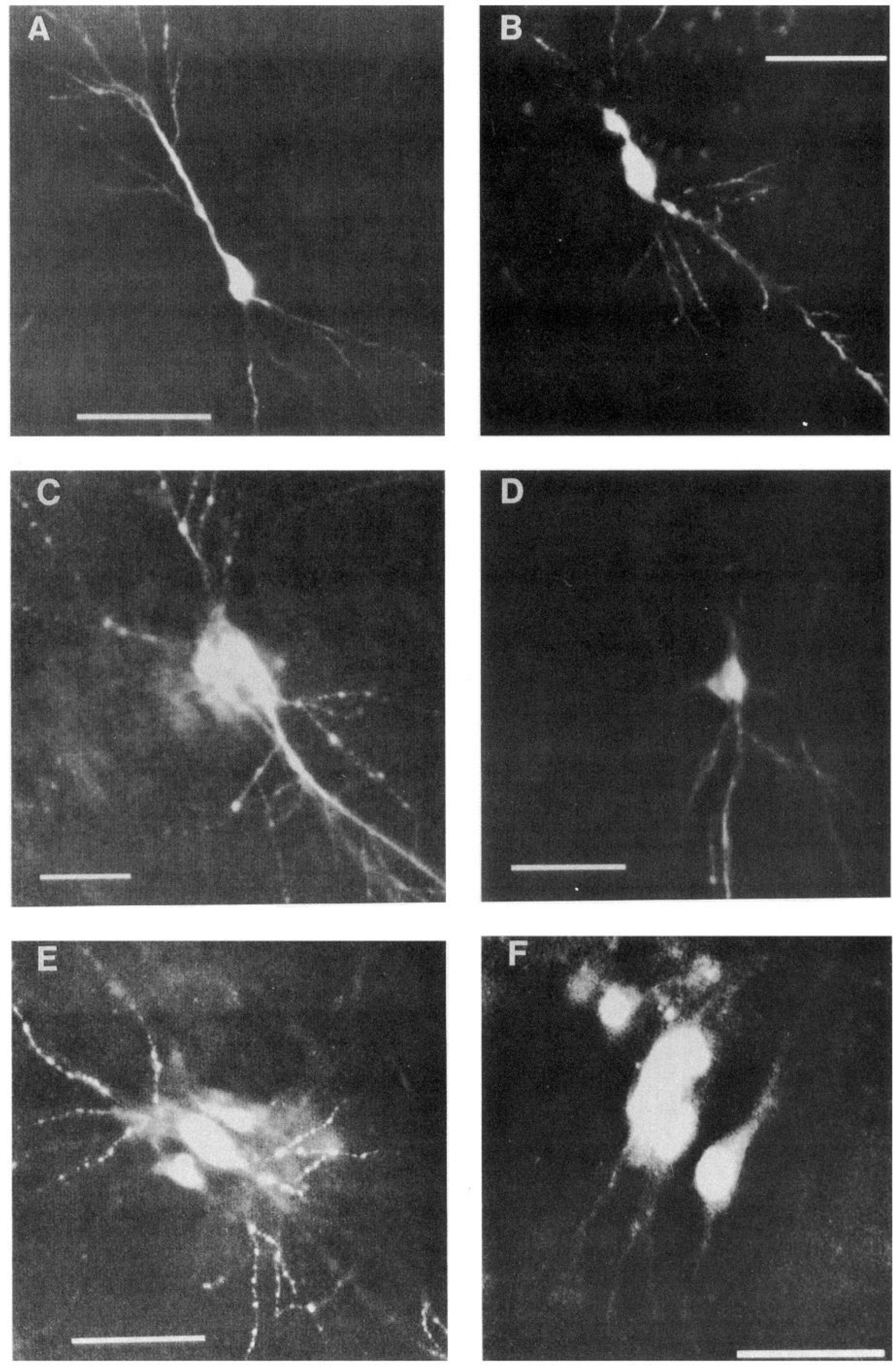
was compared with coupling in control conditions during perfusion with standard aCSF. In control conditions (normal aCSF), $15.1 \%$ pyramidal cells were coupled, while during spontaneous field burst activity in calcium-free aCSF the percentage increased to $34.2 \%$ (Table 1). Multiple coupling (more than two neurons) was never observed in control while there were three groups of three dye-coupled cells and one group of at least five dye-coupled cells in calcium-free medium. Examples of dye filled neurons can be seen in Figure 1.

The percentage of dye-coupled cells in control conditions presented here $(15.1 \%)$ is low compared with previous observations in the CAl area of the hippocampus (Andrew et al., 1982; Church and Baimbridge, 1991) and may be due to the fact that patchclamp techniques were employed here rather than intracellular electrodes for dye filling. In any case, whether intracellular or patch techniques are employed no attempt can be made to estimate absolute numbers of coupling since it is suspected that dye-coupling depends greatly on tissue preparation, recording technique, and dye used (Peinado et al., 1993). We want to emphasize the relative dye coupling ratio, comparing the percentages of coupled cells during field burst activity in calciumfree perfusate with that of control. The twofold (2.3) increase in dye coupling during field burst activity is significant and indicates that there are processes that enhance electrotonic coupling either as a direct result of the zero-calcium aCSF or secondary to seizure-like activity induced by zero calcium aCSF.

When $25 \mathrm{~mm}$ sodium propionate was added to the calciumfree aCSF to acidify the neuronal cytoplasm (see below), spontaneous field burst activity was blocked and dye coupling was reduced below control levels (Table 1).

\section{Effects of putatively altering strength of electrotonic coupling on spontaneous activity}

Since enhanced electrotonic coupling between CA1 pyramidal neurons could promote synchronization of neuronal firing, and enhanced dye coupling was observed under field burst conditions, the next question that arises is whether modification of gap junctional conductance can modify field burst activity. In order to alter electrotonic coupling through gap junctions in the CAl region of the hippocampal slice during field burst activity, we used several well-established methods that have been shown to alter dye and electrotonic coupling in the brain slice and in other preparations. These techniques include acidification and alkalinization of the intracellular space using weak acids and bases, and the gap junctional blockers halothane and octanol. Although the neuronal cytoplasm could be acidified using several weak acids added to the calcium-free aCSF, sodium propionate was employed for three reasons. First, it has been well characterized that substitution of one-fourth of the $\mathrm{NaCl}$ in the aCSF by the propionate salt leads to intracellular acidification (Roos and Boron, 1981; Sharp and Thomas, 1981; Thomas, 1984). Second, dye coupling between CA 3 neurons is abolished when slices were bathed in a sodium propionate-containing solution (MacVicar and Jahnsen, 1985). Third, the $\mathrm{pH}$ of the external aCSF did not change by substituting $25 \mathrm{~mm}$ sodium chloride by sodium propionate, which is important because ex- ternal acidification would complicate interpretation of the observations.

Intracellular acidification with $25 \mathrm{~mm}$ sodium propionate blocked all spontaneous activity in calcium-free solution in all slices tested (Figs. 2, 3) $(n=18)$. To rule out nonspecific effects of the propionate salt, we also used other methods to acidify the neuronal cytoplasm, bubbling the external solution with $90 \%$ $\mathrm{CO}_{2}$ (Gutnick and Lobel-Yaakov, 1983), or the well-known technique of acid loading with washout of ammonium chloride $\left(\mathrm{NH}_{4} \mathrm{Cl}\right)$ (Giaume and Korn, 1982; Thomas, 1984; Tolkovsky and Richards, 1987). Spontaneous extracellularly measured seizure-like activity was always blocked under these conditions ( $n$ $=13$ ). In all cases, spontaneous activity resumed within $5 \mathrm{~min}$ after washout of the acidifying solution.

If closure of gap junctions abolishes spontaneous field burst activity, then increased neuronal coupling should manifest itself as augmented spontaneous activity in the calcium-free aCSF. To test this hypothesis, several agents were added to the calcium-free aCSF to alkalinize the intracellular compartment: (1) ammonium chloride (10 $\mathrm{mm}),(2)$ trimethylamine $(10 \mathrm{~mm})$, and (3) increased bicarbonate $(60 \mathrm{~mm})$. These treatments have been demonstrated to increase intracellular $\mathrm{pH}$, dye, and/or electrotonic coupling (Spray et al., 1981, 1986; Giaume and Korn, 1982; Thomas, 1984; Church and Baimbridge, 1991). Ammonium chloride offers the advantage of presenting two phases, initial alkalinization of the cell cytoplasm followed by acidification upon washout (for the specific effects of $\mathrm{NH}_{4}{ }^{+}$in electrotonic coupling, see Giaume and Korn, 1982; Tolkovsky and Richards, 1987); therefore, one treatment allows observation of the effects of increasing and decreasing intracellular $\mathrm{pH}$ (opening and closing gap junctions, respectively) on the spontancous activity. Initial application of ammonium chloride (Fig. 2B,C), which causes intracellular alkalinization, increased the frequency and duration of spontaneous field activity, while washout (which brings about acidification) suppressed all activity, like propionate $(n=11)$. Note in Figure $2 C$ the long duration (over $1 \mathrm{~min}$ ) of the field burst event that followed $\mathrm{NH}_{4} \mathrm{Cl}$ application.

Alkalinization with trimethylamine $(n=3)$ or $60 \mathrm{~mm}$ bicarbonate ( $n=1)$ always caused an increase in frequency of spontaneous field bursts. In some cases, the intense field burst activity during perfusion with the alkalinizing solution was followed by spreading depression (see Fig. $2 C$ caption), a well-documented phenomenon that follows intense seizure activity and consists of a prolonged negative potential shift in the extracellular recording associated with an accumulation of external potassium due to paroxysmal activity (Hass and Jefferys, 1984; Yaari et al., 1986). Two more gap junctional blockers, halothane and octanol (Johnston et al., 1980; Spray et al., 1986; Terrar and Victory, 1988; Peinado et al., 1993), were tested. Figure $2 D$ illustrates that octanol $(0.2 \mathrm{~mm})$ was able to stop field burst activity $(n=4)$. The same results were obtained with the gap junction blocker halothane ( $2 \%$ in aCSF) ( $n=3$; data not shown). It is important to note that octanol did not reduce frequency of cell firing (see Table 3 , cell 3 ).

The effects of the above-mentioned agents on the spontaneous field activity may be through alterations in gap junctional con-

\footnotetext{
Figure 1. Lucifer yellow-stained CA1 pyramidal neurons in control and during field burst activity. $A$, Noncoupled neuron in control condition, slice perfused with standard aCSF. $B$, Two coupled neurons in standard aCSF. $C$ and $D$, Two coupled neurons, in slices perfused with calciumfree aCSF. $E$ and $F$, Groups of three dye-coupled neurons in calcium-free solution. Lucifer yellow $(0.1 \%$ in internal solution) was allowed to dialyze for $30 \mathrm{~min}$ using the patch-clamp technique. Scale bars: $A, D$, and $E, 100 \mu \mathrm{m} ; B, C$, and $F, 50 \mu \mathrm{m}$.
} 


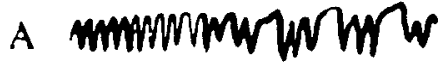

$1 \mathrm{mv}$

propionate

memprympr

wash

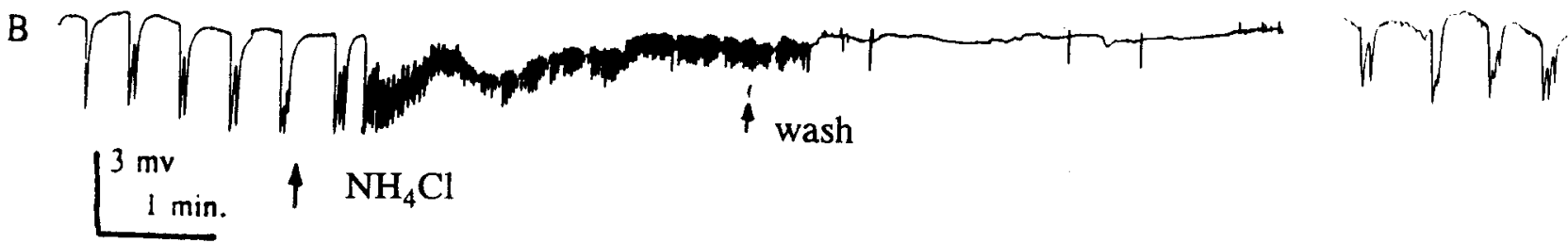

C
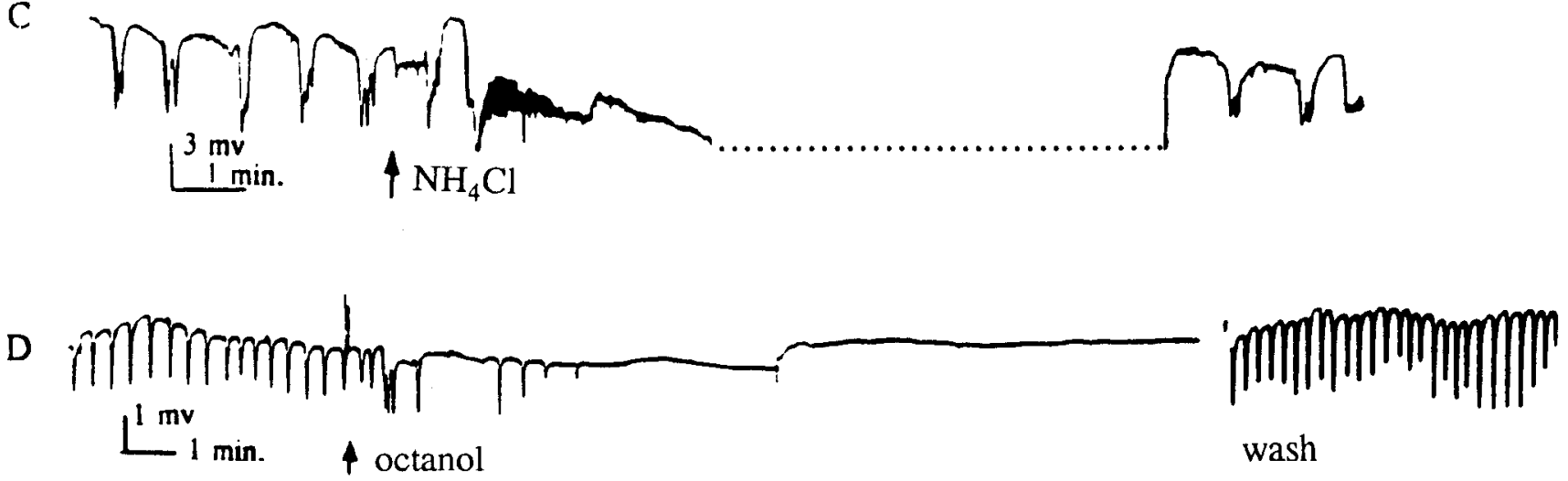

Figure 2. Effects of acidifying, alkalinizing, and octanol-containing solutions on spontaneous activity in the CA1 area. An extracellular recording electrode was placed in the cell body layer. $A$, Field bursts are suppressed in calcium-free aCSF containing 25 mm sodium propionate, which causes intracellular acidification. First trace, spontaneous activity in calcium-free aCSF; second trace, after 2 min in propionate solution. Spontaneous activity resumes within $5 \mathrm{~min}$ of washout (third trace). $B$, Field burst activity is increased upon application of calcium-free aCSF containing 10 $\mathrm{mM} \mathrm{NH}_{4} \mathrm{Cl}$ and decreased during washout. First arrow indicates change to the $\mathrm{NH}_{4} \mathrm{Cl}$-containing solution that alkalinizes intracellular compartment; note the large field burst that follows its application. Second arrow marks the change to standard calcium-free aCSF, which brings about intracellular acidification and subsequent abolition of field activity. After $5 \mathrm{~min}$, field burst activity resumes (second trace). $C$, Spreading depression caused by a large field burst upon application of $\mathrm{NH}_{4} \mathrm{Cl}$ solution. Arrow indicates start of the alkalinizing solution, which evokes a long field burst proceeded by a negative shift in the extracellularly recorded potential indicating spreading depression. The spreading depression lasted about 5 min, after which field bursting resumed. Dots indicate that the negative baseline shift of $16-17 \mathrm{mV}$ (spreading depression) is off scale. $D$, Octanol (0.2 mm) suppresses spontaneous activity. Arrow marks start of octanol-containing aCSF. Last trace was taken 8 min in washout.

ductance or through modification of intrinsic neuronal properties, in particular, action potential generation and firing frequency. To test whether this was the case, we studied the synchrony between neuronal firing and spontaneous field potential activity, for which simultaneous whole-cell and extracellular recordings were performed. In Figure 3 are typical recordings showing increased neuronal discharges in phase with the spontaneous field activity (Fig. $3 A$, left trace), while another neuron in Figure $3 B$ fires only during the field activity. These are typical situations already reported (Taylor and Dudek, 1982, 1984; Haas and Jefferys, 1984; Konnerth et al., 1986). Application of sodium propionate suppressed field activity, as shown before, but not cell firing. In fact, as shown in Figure $3 A$, this cell fires at higher frequency during application of the acidifying solution. Acidification eliminates oscillations of the neuronal membrane potential and spontaneous field activity, which presumably represents synchronized firing in a large population. Synchronized activity between field and cell firing comes back after washout of the propionate solution. Thus, acidification most likely abolishes synchronization of neuronal firing (measured as field activity), since neurons continue to fire even though the spontaneous activity has now disappeared.

Figure $3 C$ is an example of increased synchronization of spontaneous field activity and cell firing during alkalinization with $\mathrm{NH}_{4} \mathrm{Cl}$. The left trace shows poor synchrony because the neuron firing is not completely correlated with field events, while the right trace, taken 4-5 min later during application of the calcium-free/ $\mathrm{NH}_{4} \mathrm{Cl}$ aCSF, shows an increase in synchrony, the cell fires only during field events.

In order to rule out possible alterations of intrinsic cellular properties during acidification, resting membrane potential, spike amplitude and threshold, and input resistance were measured and compared with values in control (normal aCSF) and calcium-free aCSF without sodium propionate (Table 2). No significant differences in intrinsic properties during the acidification procedure were obtained. The effects of propionate were also measured in normal aCSF and, again, no differences in neuronal parameters between control aCSF and aCSF contain- 
A $\quad 5$

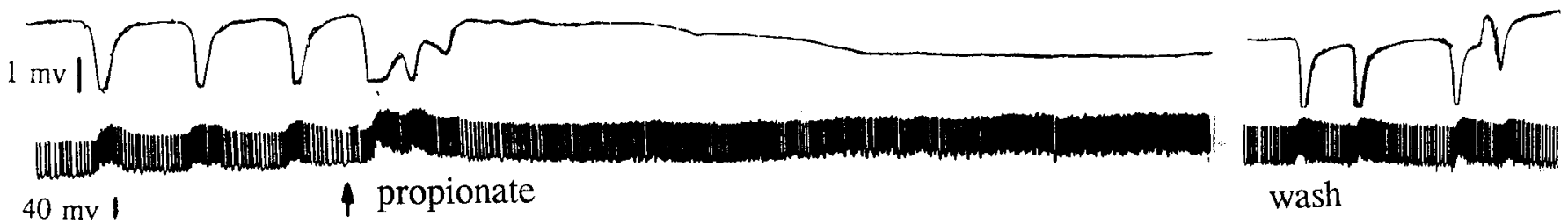

B
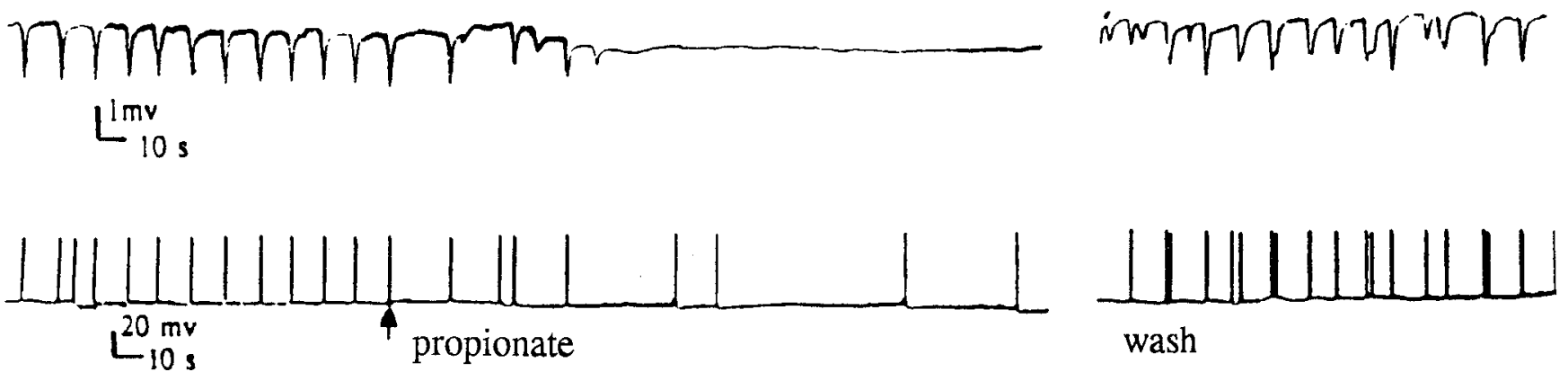

C
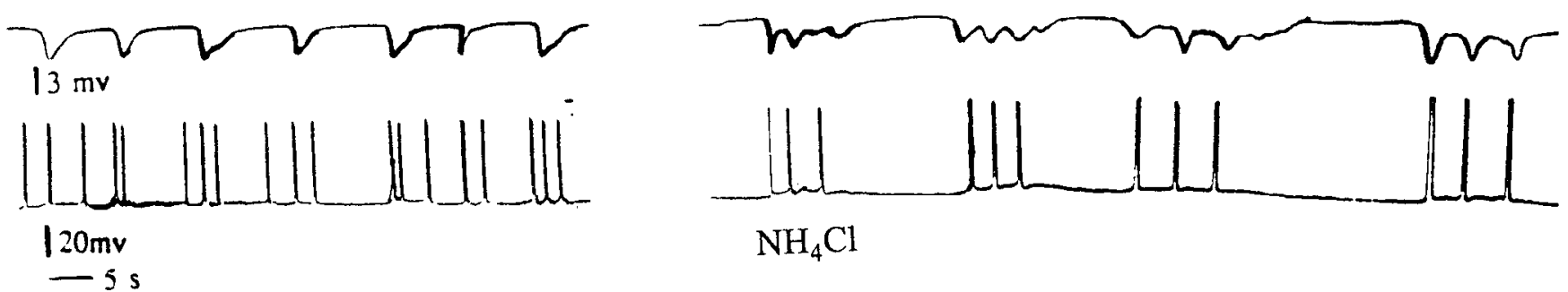

Figure 3. Simultaneous field and whole-cell recordings during field burst activity, and the effects of acidifying and alkalinizing solutions. Upper traces, field potential activity; lower traces, neuronal membrane potential. $A$ and $B$, Effects of calcium-free aCSF containing 25 mM propionate. Intracellular acidification with propionate (application begins at the arrow) abolishes synchronization between neuronal firing and spontaneous field activity. Note that the neuron fires bursts of high frequency in phase with field potential activity before application of propionate. However, the neuron fires at even higher frequency in the absence of field potential oscillations during acidification. Neuron in $B$ fires only during field activity and continues firing at random during perfusion with the acidifying solution (starts at arrow). Second traces, taken during washout, show return of synchronization between cell firing and field potential. $C$, $\mathrm{NH}_{4} \mathrm{Cl}$ further enhances synchronization between neuronal activity and negative baseline shifts in field potential (right traces) as compared to activity in calcium-free aCSF without $\mathrm{NH}_{4} \mathrm{Cl}$ (left traces). Notice that in the right traces (during $\mathrm{NH}_{4} \mathrm{Cl}$ perfusion) the cell fires only during field activity, while in the left traces, the neuron firing was not completely synchronized to the field.

ing $25 \mathrm{~mm}$ sodium propionate were apparent. It thus appears that abolition of the synchronized field burst activity through intracellular acidification is most likely mediated by a modification of gap junctional conductances, rather than alteration of intrinsic neuronal firing parameters.

\section{Acidification and octanol reduce dye coupling and fast prepotential frequency}

We have obtained evidence that acidification and alkalinization decrease and increase field burst activity, respectively, and these

Table 2. Intrinsic properties of CA1 pyramidal neurons in control, calcium-free and acidifying solution

\begin{tabular}{llll} 
& $V_{m}(\mathrm{mV})$ & $R_{\text {in }}(\mathrm{M} \Omega)$ & AP $(\mathrm{mV})$ \\
\hline Control aCSF $(n=25)$ & $-52.8 \pm 3.1$ & $99.4 \pm 23$ & $79.9 \pm 12.2$ \\
aCSF/propionate $(n=8)$ & $-51.1 \pm 1.3$ & $83.2 \pm 16.8$ & $85.3 \pm 6.5$ \\
Calcium-free aCSF $(n=21)$ & $-56.1 \pm 5.9$ & $91.5 \pm 27.2$ & $67.8 \pm 13.1$ \\
Ca-free/propionate $(n=16)$ & $-56.5 \pm 4.5$ & $96.9 \pm 13.5$ & $79.2 \pm 11.7$
\end{tabular}

Data show comparison of membrane potential $\left(V_{,}\right)$, input resistance $\left(R_{\text {in }}\right)$ and action potential amplitude (AP), measured under whole-cell configuration, of CAl pyramidal neurons in control, aCSF containing 25 mm sodium propionate, calcium-free aCSF, and same solution containing $25 \mathrm{~mm}$ propionate. Values are mean \pm standard deviation. Values are not significantly different (Student's $t$ test). 
Table 3. Frequency of FPPs and action potentials during spontaneous field activity and effects of acidification and octanol in three CA1 pyramidal neurons

\begin{tabular}{lllll} 
Cell & Condition & $\begin{array}{l}\text { FPP } \\
\text { fre- } \\
\text { quency } \\
(\mathrm{Hz})\end{array}$ & $\begin{array}{l}\text { Mean FPP } \\
\text { amplitude } \\
(\mathrm{mV})\end{array}$ & $\begin{array}{l}\text { Spike } \\
\text { fre- } \\
\text { quency } \\
(\mathrm{Hz})\end{array}$ \\
\hline $1 \quad$ Calcium-free aCSF & 5.46 & $2.18 \pm 0.4$ & 9.4 \\
& Calcium-free/propionate & 0.0 & - & 9.1 \\
& Calcium-free aCSF & 1.5 & $0.88 \pm 0.05$ & 3.5 \\
& Calcium-free/propionate & 0.38 & $0.87 \pm 0.05$ & 2.5 \\
& Propionate washout & 2.6 & $0.91 \pm 0.02$ & 2.05 \\
& Calcium-free & 0.5 & $3.43 \pm 0.1$ & 1.5 \\
& Calcium-free/propionate & 0.05 & $3.30 \pm 0.07$ & 1.5 \\
& Propionate washout & 0.43 & $3.32 \pm 0.08$ & 1.47 \\
& Calcium-free/octanol & 0.06 & $3.6 \pm 0.08$ & 1.7 \\
& Octanol washout & 0.22 & $3.52 \pm 0.09$ & 1.5 \\
\hline
\end{tabular}

Recordings made from 11 CAl pyramidal neurons revealed four that showed FPPs in calcium-free aCSF. The effects of intracellular acidification in three cells are shown. Acidifying solution containing sodium propionate $(25 \mathrm{~mm})$ or octanol $(0.2 \mathrm{~mm})$ blocked or reduced frequency of spontaneous FPPs. Third column indicates firing frequency of the three neurons under the specificd conditions. Amplitudes of FPPs are values \pm SEM.

effects were proposed to be caused through modifications of gap junctional mechanisms. To determine whether the acidification treatment was actually blocking gap junctions, we studied the number of dye-coupled pyramidal neurons in calcium-free aCSF containing propionate using Lucifer yellow as before. In these conditions, only in 1 of 23 recordings did we observe dye coupling (Table 1). Thus, $25 \mathrm{~mm}$ propionate was able to block dye coupling via gap junctions in calcium-free aCSF.

Since the time scale of the dye-filling experiments is long (cells were dialyzed with the dye for $30 \mathrm{~min}$ ) relative to the time required to establish and abolish field burst activity, we wanted to study the effects of acidification and other junctional blockers on a much shorter time scale (seconds) using an electrophysiological correlate of electrotonic coupling. For this reason, the frequency of fast prepotentials (FPPs) was quantified in calciumfree aCSF, and under conditions that presumably block gap junctions (acidification and octanol). FPPs are considered to be action potentials generated in one cell that are transmitted through gap junctions to a coupled cell. These events are rapidly decaying small stereotypic depolarizations $(1-10 \mathrm{mV}$ ) (see Fig. 4) (Spencer and Kandel, 1961; MacVicar and Dudek, 1982; Taylor and Dudek, 1982; Dudek et al., 1983; Nuñez et al., 1990; O'Donnell and Grace, 1993). Correlation between dye-coupled hippocampal pyramidal neurons and FPPs has been done by Nuñez et al. (1990) in vivo. In our experiments we found that the number of neurons displaying FPPs (36.4\%, 4 of 11) during field burst activity was similar to the incidence of dye coupling observed under similar conditions $(34.2 \%)$. The FPPs we recorded had all the characteristics previously reported in the literature, including invariance of amplitude with membrane potential. The effects of acidification and octanol can be seen in Table 3. Both treatments blocked or reduced the frequency of spontaneous FPPs in all neurons in 30-60 sec after application, but the frequency of cell firing was not altered significantly (e.g., for cell 1, spike frequency was $9.4 \mathrm{~Hz}$ in calciumfree aCSF and $9.1 \mathrm{~Hz}$ in propionate). Thus, the time course of reduction of presumed electrotonic events (FPPs) correlates well
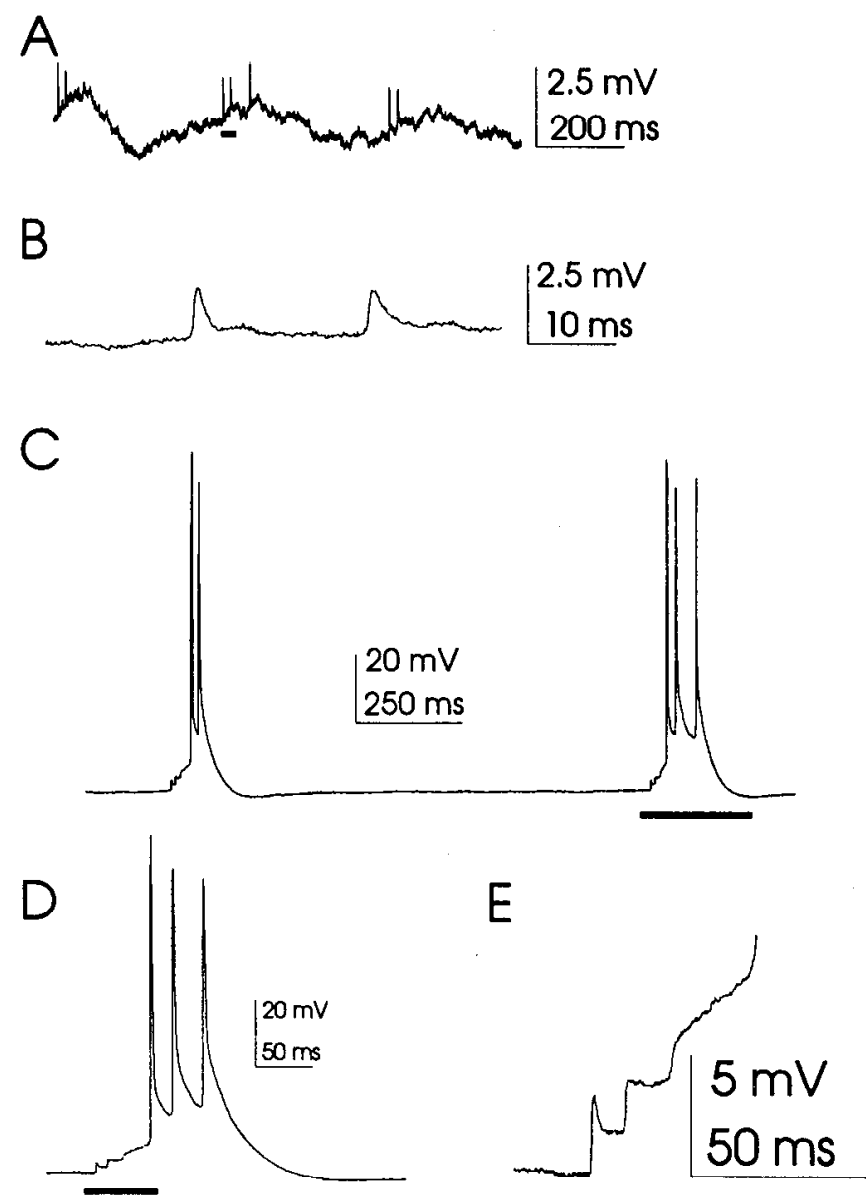

Figure 4. Fast prepotentials are correlated with intracellular spiking activity during field burst activity induced by calcium-free aCSF. $A$, Membrane potential record from a CA1 neuron hyperpolarized by 20 $\mathrm{mV}$ to prevent spiking. Note the correlation of FPPs with slow intracellular depolarizations and the similarity with Figure 6 of Nuñez et al. (1990). $B$, Expanded region marked by solid bar in $A$ illustrating FPPs in this neuron. $C$, Different neuron without applied hyperpolarizing current. Notice that a stereotyped burst of three FPPs always precedes spiking activity. This neuron only discharged after a train of FPPs. $D$ and $E$, Expanded region marked by solid bars in $C$ and $D$, respectively, to illustrate FPP summation preceding a burst of action potentials.

with the suppression of field burst activity by acidification and octanol.

\section{FPPS can synchronize neuronal firing}

In vivo experiments have revealed that FPPs are strongly correlated with the depolarizing phase of the intracellularly recorded theta rhythm (Nuñez et al., 1990). Since theta activity represents synchronous neuronal firing, during field burst activity one would expect temporal correlation between FPPs and slower intracellular oscillations; we consider slower intracellular oscillations possibly manifestations of synchronized neuronal discharge (i.e., field effects and/or extracellular potassium accumulation), and FPPs a result of "presynaptic" action potential generation phase-locked to population activity. Therefore, in a postsynaptic neuron hyperpolarized in order to suppress intrinsic action potential generation (Fig. $4 A$ ), FPPs phase-locked to slow intracellular oscillations represent "presynaptic" action potential generation in phase with synchronized population activity. In another neuron (Fig. $4 C-E$ ), the occurrence of FPPs is 
highly correlated with burst generation. This particular neuron discharged bursts of action potentials that were always preceded by a stereotyped train of three FPPs (Fig. $4 E$ ), presumably generated in a neighboring neuron and transmitted through gap junctions. It has recently been observed that FPPs can trigger action potentials in normal conditions in neurons of the nucleus accumbens (O’Donnell and Grace, 1993).

\section{Discussion}

We have presented evidence for the contribution of electrotonic interactions through gap junctions in the generation of spontaneous activity in the CAl area of the hippocampus in the calcium-free model of epilepsy. It was found that dye coupling among pyramidal neurons was increased twofold during the period of field burst activity as compared with control conditions in normal aCSF, in which there was no spontaneous field activity. Furthermore, by altering gap junctional conductances by either acidification or alkalinization of the neuronal cytoplasm, treatments that block and enhance electrotonic coupling, respectively, spontaneous activity was abolished or increased. That acidification blocked gap junctions was demonstrated by the reduction in dye-coupled neurons under conditions that suppressed spontaneous activity (i.e., propionate-containing aCSF), and by the reduction in FPP frequency in 30-60 sec after application of propionate-containing solution. Decreased spontaneous activity was also seen in the presence of two other gap junctional blockers, halothane and octanol. Octanol also blocked spontaneous FPPs in a time scale similar to propionate.

There has been a great deal of discussion regarding the relative contribution of electrotonic coupling to the synchronization of neurons during seizure-like activity. Their role as a potential mechanism of synchronization has been dismissed due to the relative scarcity of gap junctions in the mammalian brain (Haas and Jefferys, 1984). However, what should be considered is the functional manifestation of gap junctions rather than their absolute numbers. The observation that these junctions can be dynamically modulated by development, intracellular $\mathrm{pH}$, and neuronal damage (Murphy et al., 1983) makes it possible that, during epileptic seizures, electrotonic interactions are modulated by changes in $\mathrm{pH}$ that occur during intense neuronal activity (Somjen, 1984; Walz, 1989; Chesler and Kaila, 1992) and by damaged cells in epileptic foci. Gap junctions between glial cells will probably be affected also, in which case, an alteration in the removal of external potassium can be expected. Our observation that there is an increase in dye-coupled neurons during the period of spontaneous activity in the CAl area of the hippocampus suggests that electrotonic interactions are more active during seizure-like activity in the calcium-free model of epilepsy. This, together with the suppression of field burst activity upon intracellular acidification and increased frequency during alkalinization, suggests an active role for electrotonic interactions in synchronizing neuronal firing.

Interestingly, it was reported by Church and Baimbridge (1991) that the number of dye-coupled CA1 pyramidal neurons doubled when slices were exposed to an alkalinizing solution. Furthermore, dye-coupled neurons exhibited a characteristic burst firing pattern, which could reflect localized field effects reinforced by electrotonic coupling. Since we saw a similar increase in coupled neurons (2.3 times) with zero-calcium perfusate, it could be that transient changes in $\mathrm{pH}$, specifically the alkaline shift that occurs during paroxysmal activity (Walz, 1989; Chen and Chesler, 1992; Chesler and Kaila, 1992), or decreased in- tracellular calcium, are responsible for the enhanced coupling during epileptiform activity. In any case, our purpose was not to investigate the mechanisms by which gap junctions are modulated, but to determine whether there was a change in neuronal coupling during spontaneous activity.

As has been discussed by Dudek et al. (1983), it is unlikely that in the mammalian brain there is extensive and strong coupling among neuronal populations. This has been indirectly confirmed by numerous dye-filling experiments showing coupling among small groups of neurons rather than extensive syncytia (see also Fig. 1), as is the case for glial elements. In conditions of calcium-free medium that enhance dye coupling, restricted dye coupling was still observed, although more extensive than in control conditions. From a functional perspective, the electrophysiological correlate of electrotonic coupling, the FPPs, could synchronize activity of neighboring neurons, presumably those in a coupled group (e.g., see Fig. $4 C-E$ ). In Figure $4 D$, a repetitive pattern of FPPs and action potentials was recorded, with three superimposed FPPs (temporal summation) followed by neuronal firing after the third. If the spike threshold was higher, as in standard aCSF with $2 \mathrm{mM} \mathrm{CaCl}_{2}$, these FPPs would not have caused cellular discharge. Thus, electrotonic coupling can become extremely important in firing synchronization when neurons are near firing threshold, since the gain of the system is infinitely high (Bennett, 1977). For example, in lower vertebrate preparations, weakly coupled neurons fire in synchrony if the group is near firing threshold (Bennett, 1977; Dudek et al., 1986). In calcium-free solution, neurons become very excitable for several reasons, including lack of calcium-activated potassium conductances, like $I_{C}$ involved in spike repolarization (Storm, 1987) and $I_{\mathrm{AHP}}$, involved in spike frequency adaptation (Lancaster and Adams, 1986), and their firing thresholds are lower due to a lack of screening charges (Frankenhauser and Hodgkin, 1957). Therefore, any mechanism that enhances electrotonic coupling will act as a feedback mechanism, causing coupled neurons to fire in synchrony. This is similar to the concept of burst propagation mediated by chemical synapses in the disinhibited CA3 region (Miles and Wong, 1987), albeit through electrotonic synapses rather than chemical synapses. Thus, during field burst activity the functional unit effectively becomes each small neuronal aggregate, rather than individual neurons. Each aggregate can then potentially affect the neighboring aggregate, since ionic and electrical gradients established by a bursting group of neurons would be greater than those generated by any one neuron. Propagation of activity through the pyramidal layer would then be expected to be saltatory (from aggregate to aggregate), but still limited by the generation of electrical and ionic gradients. Computer simulation studies, which quantitate effects of electrotonic interactions in promoting synchronization and oscillation of neuronal potentials, are under way in our laboratory.

It then appears that during seizure-like activity there could be a feedback mechanism involving at least three factors: increased extracellular concentration of potassium, ephaptic interactions, and electrotonic coupling. Each one is necessary but not sufficient for triggering synchronized spontaneous activity. Alterations in potassium gradients appear important, since small changes in potassium concentration can alter field burst activity (Yaari et al., 1986; Schweitzer et al., 1992). Electrical fields applied to the hippocampal slice can also change this activity (Kayyali and Durand, 1991), and here we have obtained evidence that altering gap junctional conductance can suppress or 
increase spontaneous activity. Thus, our results and those of others (see, e.g., Spray et al., 1981, 1986, on the rapid effects of $\mathrm{pH}$ changes on junctional conductance) suggest that electrotonic coupling can be modified rapidly over a time scale of seconds. We cannot say at the present time whether this alteration of gap junctional mechanisms is due to changes in $\mathrm{pH}$ and/or calcium levels, but it is very likely that both factors will contribute to alter junctional conductances.

Evidence that gap junctions could play an important role in human epilepsies was reported by Naus et al. (1991), where the levels of mRNA for connexins (proteins that form gap junctions) were found to be elevated in tissue from epileptic patients, suggesting that gap junction proteins are overexpressed in these pathologies (in neurons and probably glial cells). Furthermore, hyperventilation for 3-5 min (resulting in a respiratory alkalosis) is most effective at triggering epileptic absence attacks, but generally may enhance any epileptic event (Pedley, 1984).

In summary, our observations indicate that gap junctional mechanisms are altered during field burst activity in the CA1 region induced by calcium-free aCSF, and suggest that electrotonic interactions can participate in synchronizing neuronal firing during seizure-like activity. In neocortex it appears that neuronal dye coupling is more extensive in young animals than more mature ones (Peinado et al., 1993). Since the animals employed in these studies were relatively young (20-30 d old), it is possible that the potential role of gap junctional mechanism in neuronal synchronization (as described here) would be reduced in the case of adult animals, where there is not as much coupling as in young animals. The relevance of these results obtained in this in vitro epilepsy model to epileptic pathologies in humans remains to be determined, with the optimistic possibility that the cellular and network bases of seizure activity in vivo is a superset of mechanisms including those involved in this calcium-free model of seizures.

\section{References}

Andrew RD, Taylor CP, Snow RW, Dudek FE (1982) Coupling in rat hippocampal slices: dye transfer between CA1 pyramidal cells. Brain Res Bull 8:211-222.

Baimbridge KG, McLennan PMJ, Church J (1991) Bursting response to currents-evoked depolarization in rat CAl pyramidal neurons is correlated with Lucifer Yellow dye coupling but not with the presence of calbindin-D28k. Synapse 7:269-277.

Bennet MVL (1977) Electrical transmission: a functional analysis and comparison to chemical transmission. In: Handbook of physiology, Sect I, The nervous system, Vol 1, Pt 1 (Kandel ER, ed), pp 357416. Bethesda, MD: American Physiological Society.

Chen JCT, Chesler M (1992) Extracellular alkaline shifts in rat hippocampal slice are mediated by NMDA and non-NMDA receptors. J Neurophysiol 68:342-344.

Chesler M, Kaila K (1992) Modulation of pH by neuronal activity. Trends Neurosci 15:396-402.

Church J, Baimbridge K (1991) Exposure to high-pH medium increases the incidence and extent of dye coupling between rat hippocampal CA1 pyramidal neurons in vitro. J Neurosci 11:3289-3295.

Dermietzel R, Spray DC (1993) Gap junctions in the brain: where, what type, how many and why?. Trends Neurosci 16:186-192.

Dichter MA (1989) Cellular mechanisms of epilepsy and potential new treatment strategies. Epilepsia 30:S3-S12.

Dudek FE, Andrew RD, MacVicar BA, Snow RW, Taylor CP (1983) Recent evidence for and possible significance of gap junctions and electrotonic synapses in the mammalian brain. In: Basic mechanisms of neuronal hyperexcitability, pp 31-73. New York: Liss.

Dudek FE, Snow RW, Taylor CP (1986) Role of electrical interactions in synchronization of epileptiform bursts. In: Advanccs in ncurology, Vol 44 (Delgado Escueta AV, Ward AA, Woodbury DM, Porter RJ, eds), pp 593-617. New York: Raven.
Frankenhaeuser B, Hodgkin AL (1957) The action of calcium on the electrical properties of squid axon. J Physiol (Lond) 137:218-244.

Giaume C, Korn H (1982) Ammonium sulfate induced uncouplings of crayfish axons with and without increased junctional conductance. Neuroscience 7:1723-1730.

Gutnick MJ, Lobel-Yaakov R (1983) Carbon dioxide uncouples dye coupled neuronal aggregates in neocortical slices. Neurosci Letl 42: 197-200.

Haas HL, Jefferys JGR (1984) Low-calcium field burst discharges of CA1 pyramidal neurones in rat hippocampal slices. J Physiol (Lond) 354:185-201

Hamill OP, Marty A, Neher A, Sakmman B, Sigworth FJ (1981) Improved patch-clamp techniques for high-resolution recording from cells and cell-free membrane patches. Pfluegers Arch 391:85-100.

Johnston MF, Simon SA, Ramon F (1980) Interactions of anaesthetics with electrical synapses. Nature 286:498-500.

Kayyali H, Durand D (1991) Effects of applied currents on epileptiform bursts in vitro. Exp Neurol 113:249-254.

Konnerth A, Heinemann U, Yaari Y (1986) Nonsynaptic epileptogenesis in the mammalian hippocampus in vitro. I. Development of seizurelike activity in low extracellular calcium. J Neurophysiol 56: 409-421.

Lancaster B, Adams PR (1986) Calcium-dependent current generating the afterhyperpolarization of hippocampal neurons. J Neurophysiol 55:1268-1282.

Lazrac A, Peracchia C (1993) Gap junction gating sensitivity to physiological internal calcium regardless of $\mathrm{pH}$ in Novikoff hepatoma cells. Biophysiol J 65:2002-2012.

MacVicar BA, Dudek FE (1981) Electrotonic coupling between pyramidal cells: a direct demonstration in rat hippocampal slices. Science 213:782-785.

MacVicar BA, Dudek FE (1982) Electrotonic coupling between granule cells of rat dentate gyrus: physiological and anatomical evidence. J Neurophysiol 47:579-592.

MacVicar BA, Jahnsen H (1985) Uncoupling of CA3 pyramidal neurons by propionate. Brain Res 330:141-145.

Miles R, Wong RKS (1987) Inhibitory control of local excitatory circuits in the guinea-pig hippocampus. J Physiol (Lond) 388:611629

Murphy AD, Hadley RD, Kater SB (1983) Axotomy-induced parallel increases in electrical and dye coupling between identified neurons of Helisoma. J Neurosci 3:1422-1429.

Naus CCG, Bechberger JF, Paul DL (1991) Gap junction gene expression in human seizure disorder. Exp Neurol 111:198-203.

Nuñez A, García Austt E, Buño W (1990) In vivo electrophysiological analysis of Lucifer Yellow coupled hippocampal pyramids. Exp Neurol 108:76-82.

O'Donnell P, Grace AA (1993) Dopaminergic modulation of dye coupling between neurons in the core and shell regions of the nucleus accumbens. J Neurosci 13:3456-3471.

Pedley TA (1984) Epilepsy and the human electroencephalogram. In: Electrophysiology of epilepsy (Schwartzkroin PA, Wheal HV, eds), pp 1-30. London: Academic.

Peinado A, Yuste R, Katz LC (1993) Extensive dye coupling between rat neocortical neurons during the period of circuit formation. Neuron 10:103-114

Roos A, Boron WF (1981) Intracellular pH. Physiol Rev 61:296-434.

Schmalbruch H, Jahnsen H (1981) Gap junctions on CA3 pyramidal cells of guinea-pig hippocampus shown by freeze fracture. Brain Res 217:175-178.

Schweitzer JS, Patrylo PR, Dudek FE (1992) Prolonged field bursts in the dentate gyrus: dependence on low calcium, high potassium, and nonsynaptic mechanisms. J Neurophysiol 68:2016-2025.

Sharp AP, Thomas RC (1981) The effects of chloride substitution on intracellular $\mathrm{pH}$ in crab muscle. $\mathrm{J}$ Physiol (Lond) 312:71-80.

Shiosaka S, Yamamoto T, Hertzberg EL, Nagy JI (1989) Gapjunction protein in rat hippocampus: correlative light and electron microscope immunohistochemical localization. J Comp Neurol 281:282-297.

Snow RW, Dudek FE (1984) Electrical fields directly contribute to action potential synchronization during convulsant-induced epileptiform bursts. Brain Res 323:114-118.

Somjen GG (1984) Acidification of interstitial fluid in hippocampal formation caused by seizures and spreading depression. Brain Res 311:186-188.

Spencer WA, Kandel ER (1961) Electrophysiology of hippocampal neurons. IV. Fast prepotentials. J Neurophysiol 24:272-285. 
Spray DC, Harris AL, Bemet MVL (1981) Gap junctional conductance is a simple and sensitive function of intracellular $\mathrm{pH}$. Science 211:712-715.

Spray DC, Ginzberg RD, Morales EA, Gatmaitan Z, Arias IM (1986) Electrophysiological properties of gap junctions between dissociated pairs of rat hepatocytes. J Cell Biol 103:135-144.

Stewart WW (1981) Lucifer dyes-highly fluorescent dyes for biological tracing. Nature 292:17-21.

Storm JF (1987) Action potential repolarization and a fast after-hyperpolarization in rat hippocampal pyramidal cells. J Physiol 385: 733-759.

Taylor CP, Dudek FE (1982a) Synchronous neural afterdischarges in rat hippocampal slices without active chemical synapses. Science 218 : 810-812.

Taylor CP, Dudek FE (1982b) A physiological test for electrotonic coupling between CA1 pyramidal cells in rat hippocampal slices. Brain Res 235:351-357.

Taylor CP, Dudek FE (1984) Synchronization without active chemical synapses during hippocampal afterdischarges. J Neurophysiol 52:145155.
Terrar DA, Victory JGG (1988) Influence of halothane on electrical coupling in cell pairs isolated from guinea-pig ventricle. Br J Pharmacol 94:509-514.

Thomas RC (1984) Experimental displacement of intracellular $\mathrm{pH}$ and the mechanisms of its subsequent recovery. J Physiol (Lond) 354: $3-22$.

Tolkovsky AM, Richards CD (1987) $\mathrm{Na}^{+} / \mathrm{H}^{+}$exchange is the major mechanism of $\mathrm{pH}$ regulation in cultured sympathetic neurons: measurements in single cell bodies and neurites using a fluorescent $\mathrm{pH}$ indicator. Neuroscience 22:1093-1102.

Traub RD, Miles R, Wong RK (1989) Model of the origin of rhythmic population oscillations in the hippocampal slice. Science 243:13191324.

Walz W (1989) pH shifts evoked by neuronal stimulation in slices of rat hippocampus. Can J Physiol Pharmacol 67:577-581.

Yaari Y, Konnerth A, Heinemann U (1986) Nonsynaptic epileptogenesis in the mammalian hippocampus in vitro. II. Role of extracellular potassium. J Neurophysiol 56:424-437. 